\title{
Light-Activated Staudinger-Bertozzi Ligation within Living Animals
}

\author{
Lisa Shah ${ }^{\dagger}$, Scott T. Laughlin ${ }^{\dagger, \ddagger}$, and Isaac S. Carrico ${ }^{\dagger, \ddagger},{ }^{*}$ \\ tDepartment of Chemistry, State University of New York Stony Brook, Stony Brook, New York \\ 11794-3400, United States \\ FInstitute of Chemical Biology and Drug Discovery, State University of New York Stony Brook, \\ Stony Brook, New York 11794-3400, United States
}

\section{Abstract}

The ability to regulate small molecule chemistry in vivo will enable new avenues of exploration in imaging and pharmacology. However, realization of these goals will require reactions with high specificity and precise control. Here we demonstrate photocontrol over the highly specific Staudinger-Bertozzi ligation in vitro and in vivo. Our simple approach, photocaging the key phosphine atom, allows for the facile production of reagents with photochemistry that can be engineered for specific applications. The resulting compounds, which are both stable and efficiently activated, enable the spatial labeling of metabolically introduced azides in vitro and on live zebrafish.

The use of bioorthogonal chemical reporters to tag naturally occurring biomolecules in their native setting is a powerful tool for studying and manipulating biological processes. ${ }^{1-4}$ Azides are particularly potent in this setting as they are small, stable under physiological conditions, easy to access synthetically, and generally well tolerated by biosynthetic machinery. ${ }^{5}$ These attributes have led to the widespread incorporation of azides into biological molecules, which in turn has spurred the transformation of bioorthogonal chemistry over the past 15 years. Novel azide selective chemistries developed during this time frame include the Staudinger-Bertozzi ligation, ${ }^{6}$ copper-catalyzed "click" chemistry, and strain-promoted "click" chemistry. ${ }^{8-10}$

The Staudinger-Bertozzi ligation, commonly utilized in vivo, has the advantages of not requiring a catalyst, forming amide bonds, and exhibiting exquisite chemoselectivity. Despite slower kinetics, Staudinger-Bertozzi probes have outperformed strain-promoted cycloaddition probes in vivo. ${ }^{11}$ These findings may reflect the higher metabolic stability of phosphine reagents in contrast to strained alkynes. While faster strain-promoted reagents

\footnotetext{
*Corresponding Author. isaac.carrico@stonybrook.edu. ASSOCIATED CONTENT Supporting Information The Supporting Information is available free of charge on the ACS Publications website at DOI: 10.1021/jacs.5b13401.

Experimental details and data (PDF)
} 
have emerged, the Staudinger-Bertozzi ligation remains widely utilized. Attempts at enhancing the kinetics of the reaction by increasing the electron density on the phosphine have been somewhat successful, but have not translated well in vivo due to premature oxidation of the phosphine. ${ }^{12,13}$ Despite these limitations, the Staudinger-Bertozzi Ligation is an indispensible tool for bioorthogonal labeling in vitro and in vivo.

The biological utility of a number of bioorthogonal reactions has been expanded through the development of photoactivatable variants, which enables spatial and temporal control of target labeling. ${ }^{14-17}$ In this context, a photocleavable protecting group can be covalently attached to a critical functional group on a given molecule, effectively inactivating it. Upon exposure at the appropriate wavelength of light, the protecting group is removed to reveal the reactive, parent molecule. A previous study by the Popik group involving a phototriggered dibenzocyclooctyne was shown to label azide-bearing glycoproteins on $\mathrm{CHO}$ cells through the strain-promoted click reaction. ${ }^{18}$ However, complicated synthesis of cyclopropenone-masked dibenzocyclooctynes and low wavelengths required for photoactivation have limited their widespread utility in vivo.

Recently, fluorogenic two-photon-activated naphthalene-based tetrazoles have been reported for a phototriggered nitrile imine cycloaddition. ${ }^{19}$ The most noteworthy feature of these probes is the ability to photoactivate using wavelengths in the near-infrared range which, coupled with the fast kinetics of the bioorthogonal reaction, certainly facilitates their in vivo use. However, lengthy and complex synthesis of these photocaged precursors has likely limited their utility. While this reaction is relatively fast in vitro, inhibition by chloride significantly reduces labeling efficiency in cells. In both examples, no measure of photoefficiency, critical for in vivo utility, was reported.

Here, we describe a caged Staudinger-Bertozzi reagent that can be efficiently photoactivated to yield a reactive phosphine. Photocaged phosphines such as 1a are not susceptible to oxidation (in contrast to the parent triarylphosphine) and do not react with azides prior to removal of the photoprotecting group. We demonstrate that photocaged phosphines can be used to spatially label azide-bearing glycoproteins on mammalian cells and on fixed zebrafish larvae. Notably, these reagents are modular and easy to synthesize. Due to the inherent modularity and generality, activation wavelengths, reporter tags, and protein engineering modifications are all programmable, which is expected to markedly increase the utility of these reagents, particularly with respect to in vivo applications.

A range of 4,5-dimethoxy-2-nitrobenzyl (DMNB)-caged phosphines can be straightforwardly obtained via nucleophilic attack of commercially available DMNB bromide by the appropriate triarylphosphine (see Supporting Information (SI)). As there is little literature precedence for the photouncaging of phosphines, initial experiments focused on their stability, toxicity, and DMNB release efficiency (Figures S1 and S2). Particularly, we were concerned that uncaging of 1a might accelerate phosphine oxidation and reduce the amount of reactive phosphine available for ligation. We estimated the productivity of this reaction indirectly by adding azide to a solution of the caged small molecule to monitor the efficiency of the resulting Staudinger-Bertozzi ligation reaction. An initial sample containing $6.5 \mathrm{mM} \mathrm{1a}$ and $10.0 \mathrm{mM} 2$ was irradiated with UV light. LC-UV-MS analysis of 
samples before and after light exposure allowed us to identify and quantify the products formed. By comparing the ratio of the chromatographic areas (by UV absorbance) of the two possible ligation products (ligated product $\mathbf{3}$ and phosphine oxide $\mathbf{5}$ ), the uncaging reaction efficiency was calculated to be $86 \% \pm 10 \%$ (Figure 1). Though this is an estimated value due to the inherent complexity of analysis, these results indicate that an overwhelming majority of the desired product remains unoxidized following uncaging. Notably, phosphine deprotection is $~ 1.5$-fold slower than DMNB-caged thiols (Figure S3), which was expected given mechanistic investigations of $o$-nitrobenzyl-type photoprotecting groups and the effects of various leaving groups on photolysis quantum yields and rates. ${ }^{20}$ Additionally, the quantum yield of DMNB-caged phosphines was determined to be $\Phi=0.03$ (see SI). A literature survey of $o$-nitrobenzyl-caged compounds reveals that these values fall within previously reported photochemical parameters previously shown by others for caged alcohols, esters, and ethers (reported QY range from 0.0075 to 0.7 ). ${ }^{21}$

In order to establish the utility of the photocaged probe in a complex, biologically relevant setting, cell lysates containing azide-bearing glycoproteins were labeled using an epitopemodified phosphine. The synthesized reporter-conjugated caged phosphine, $\mathbf{1 b}$, allowed for the detection of metabolically labeled glycoproteins from the HEK293 cell lysate via Western blot (Figure 2a). HEK293 cells were cultured in the presence of the sialic acid precursor $\mathrm{N}$-azidoacetylmannosamine ( $\mathrm{Ac}_{4} \mathrm{ManNAz}$ ) for 3 days to metabolically incorporate $\mathrm{N}$-azidoacetyl-sialic acid (SiaNAz) residues into cell-surface glycoproteins. ${ }^{10}$ Cells cultured in the absence of azidosugar were used as a negative control. Whole-cell lysates extracted from the cells described above were incubated with $500 \mu \mathrm{M}$ of $\mathbf{1 b}$ or phosphine-FLAG as a control. $^{22}$ UV-irradiated samples were exposed between 30 and $180 \mathrm{~min}$. Samples were separated by SDS-PAGE and immunostained with an anti-FLAG M2-HRP antibody. As expected, Western blot analysis demonstrated that labeling with 1b was UV light dependent, and increased UV exposure time led to a commensurate increase in signal (Figure 2b). A similar experiment was performed on $\mathrm{N}$-azidoacetylgalactosamine (GalNAz)-labeled adenovirus type 5 to characterize the light-dependent labeling of a single, identifiable protein (Figure S6).

One of the primary advantages of photocaging is the unique ability to spatially activate a given reaction using focused light. To assess whether a caged small molecule phosphine could be used to spatially label azidoglycoproteins, metabolically labeled HEK293 cells were fixed and treated with $300 \mu \mathrm{M} \mathbf{1 b}$ or phosphine-FLAG. After localized irradiation, within a region of interest (ROI), cells were left unperturbed for $1 \mathrm{~h}$. Subsequent immunostaining with anti-FLAG-FITC revealed that cells within the defined ROI exhibited significantly higher labeling (Figure 3a). Notably very little spread in the signal was observed. Given the kinetics of the Staudinger ligation $\left(k_{2}=2.5 \times 10^{-3} \mathrm{M}^{-1} \mathrm{~s}^{-1}\right),{ }^{13}$ some diffusion from the site of decaging is expected. Presumably these data indicate some limitation of diffusion of the decaged phosphine, perhaps due to the surface interaction of these highly charged peptides.

To establish the utility of the photocaged phosphine in vivo, zebrafish, metabolically labeled with azidosugar, were spatially labeled through focused irradiation. Embryos were injected with $5 \mathrm{~nL}$ of $5 \mathrm{mM} \mathrm{GalNAz}$ at the one-cell stage. At $72 \mathrm{~h}$ postfertilization (hpf), embryos 
were anesthetized, partially mounted in agarose, and incubated with either $5.0 \mathrm{mM}$ of $\mathbf{1 b}$ or phosphine-FLAG. The jaw region was selected as the ROI as it had previously demonstrated intense azidoglycan labeling and low levels of autofluorescence. ${ }^{9}$ Embryos were subsequently fixed and immunostained with an anti-FLAG-FITC antibody for visualization via fluorescence microscopy (Figure 3b). As expected, fluorescent signal was limited to the irradiated area, although there is significantly more spread than was observed in vitro.

In summary, we have synthesized photocaged Staudinger-Bertozzi reagents that efficiently uncage to produce reactive phosphines, which can be used to spatially modify azides on cell surfaces (both in vitro and in vivo). To the best of our knowledge, this is the first example of a photoinitiated bioorthogonal ligation within a living organism. These reagents are stable and efficiently photoactivated in vivo. While the bioorthogonal ligation rate limits the biological applicability to slower time scale dynamics, one of the primary advantages of our technique is its synthetic flexibility in the choice of photocage (see SI, compound 6) and labeling probe (see Figure S7, 1c), which allows it to be tailored for specific in vivo applications by chemists and biologists alike.

\section{Supplementary Material}

Refer to Web version on PubMed Central for supplementary material.

\section{Acknowledgments}

We thank Dr. Bela Ruzsicska and the Institute of Chemical Biology and Drug Discovery for assistance with lowresolution mass spectrometry analysis, Dr. James Marecek for assistance with NMR acquisition and analysis, Yanjie Chu for the synthesis of Ac4ManNAz, the Stony Brook University Department of Pharmacology for the use of their microscope facilities, and the Stony Brook University Proteomics Center for high-resolution mass spectrometry data (NIH/NCRR 1 S10 RR023680-1). This work was supported in part by the U.S. National Science Foundation (NSF) (CBET 1080909 to I.S.C.) and the U.S. National Institutes of Health (NIH) (T32GM092714 to L.S.)

\section{REFERENCES}

1. Swarts BM, Holsclaw CM, Jewett JC, Alber M, Fox DM, Siegrist MS, Leary JA, Kalscheuer R, Bertozzi CR. J. Am. Chem. Soc. 2012; 134:16123. [PubMed: 22978752]

2. Carroll L, Boldon S, Bejot R, Moore JE, Declerck J, Gouverneur V. Org. Biomol. Chem. 2011; 9:136. [PubMed: 21103523]

3. Dieterich DC, Link AJ, Graumann J, Tirrell DA, Schuman EM. Proc. Natl. Acad. Sci. U. S. A. 2006; 103:9482. [PubMed: 16769897]

4. Devaraj NK, Hilderbrand S, Upadhyay R, Mazitschek R, Weissleder R. Angew. Chem. Int. Ed. 2010; 49:2869.

5. (a) Luchansky SJ, Hang HC, Saxon E, Grunwell JR, Yu C, Dube DH, Bertozzi CR. Methods Enzymol. 2003; 362:249. [PubMed: 12968369] (b) Smith MD, Gong D, Sudhahar CG, Reno JC, Stahelin RV, Best MD. Bioconjugate Chem. 2008; 19:1855.(c) Grammel M, Hang HC. Nat. Chem. Biol. 2013; 9:475. [PubMed: 23868317]

6. (a) Staudinger H, Meyer J. Helv. Chim. Acta. 1919; 2:635.(b) Kiick KL, Saxon E, Tirrell DA, Bertozzi CR. Proc. Natl. Acad. Sci. U. S. A. 2002; 99:19. [PubMed: 11752401]

7. (a) Kolb HC, Finn MG, Sharpless KB. Angew. Chem. Int. Ed. 2001; 40:2004.(b) Rodionov VO, Presolski S, Díaz DD, Fokin VV, Finn MG. J. Am. Chem. Soc. 2007; 129:12705. [PubMed: 17914817] (c) Hong V, Steinmetz NF, Manchester M, Finn MG. Bioconjugate Chem. 2010; 21:1912.

8. Dehnert KW, Beahm BJ, Huynh TT, Baskin JM, Laughlin ST, Wang W, Wu P, Amacher SL, Bertozzi CR. ACS Chem. Biol. 2011; 6:547. [PubMed: 21425872] 
9. Dehnert KW, Baskin JM, Laughlin ST, Beahm BJ, Naidu NN, Amacher SL, Bertozzi CR. ChemBioChem. 2012; 13:353-357. [PubMed: 22262667]

10. Baskin JM, Prescher JA, Laughlin ST, Agard NJ, Chang PV, Miller IA, Lo A, Codelli JA, Bertozzi CR. Proc. Natl. Acad. Sci. U. S. A. 2007; 104:16793. [PubMed: 17942682]

11. Agard NJ, Baskin JM, Prescher JA, Lo A, Bertozzi CR. ACS Chem. Biol. 2006; 1:644. [PubMed: 17175580]

12. Saxon E, Bertozzi CR. Science. 2000; 287:2007. [PubMed: 10720325]

13. Lin FL, Hoyt HM, van Halbeek H, Bergman RG, Bertozzi CR. J. Am. Chem. Soc. 2005; 127:2686. [PubMed: 15725026]

14. Pauloehrl T, Delaittre G, Winkler V, Welle A, Bruns M, Borner HG, Greiner AM, Bastmeyer M, Barner-kowollik C. Angew. Chem. Int. Ed. 2012; 51:1071.

15. Adzima BJ, Tao Y, Kloxin CJ, DeForest CA, Anseth KS, Bowman CN. Nat. Chem. 2011; 3:256. [PubMed: 21336334]

16. Taniguchi A, Skwarczynski M, Sohma Y, Okada T, Ikeda K, Prakash H, Mukai H, Hayashi Y, Kimura T, Hirota S, Matsuzaki K, Kiso Y. ChemBioChem. 2008; 9:3055. [PubMed: 19025862]

17. Lim RK, Lin Q. Acc. Chem. Res. 2011; 44:828. [PubMed: 21609129]

18. Poloukhtine AA, Mbua NE, Wolfert MA, Boons GJ, Popik VV. J. Am. Chem. Soc. 2009; 131:15769. [PubMed: 19860481]

19. (a) Song W, Wang Y, Lin Q. J. Am. Chem. Soc. 2008; 130:9654. [PubMed: 18593155] (b) Yu Z, Ohulchanskyy TY, An P, Prasad PN, Lin Q. J. Am. Chem. Soc. 2013; 135:16766. [PubMed: 24168622]

20. (a) Holmes CP. J. Org. Chem. 1997; 62:2370. [PubMed: 11671569] (b) Pelliccioli AP, Wirz. Photochem. Photobiol. Sci. 2002; 1:441. [PubMed: 12659154] (c) Kim MS, Diamond SL. Bioorg. Med. Chem. Lett. 2006; 16:4007. [PubMed: 16713258] (d) Solomek T, Mercier S, Bally T, Bochet CG. Photochem. Photobiol. Sci. 2012; 11:548. [PubMed: 22237825]

21. (a) Dantzig JA, Walker JW, Trentham DR, Goldman YE. Proc. Natl. Acad. Sci. U. S. A. 1988; 85:6716. [PubMed: 3413119] (b) Klan P, Solomek T, Bochet CG, Blanc A, Givens R, Rubina M, Popik V, Kostikov A, Wirz J. Chem. Rev. 2013; 113:119. [PubMed: 23256727]

22. Laughlin ST, Bertozzi CR. Nat. Protoc. 2007; 2:2930. [PubMed: 18007630] 
a) 4

0<smiles>[CH]=CC</smiles>

\section{1}

b) 1

\section{2}

\section{3}

\section{4}

\section{5}

6

\section{7}

3

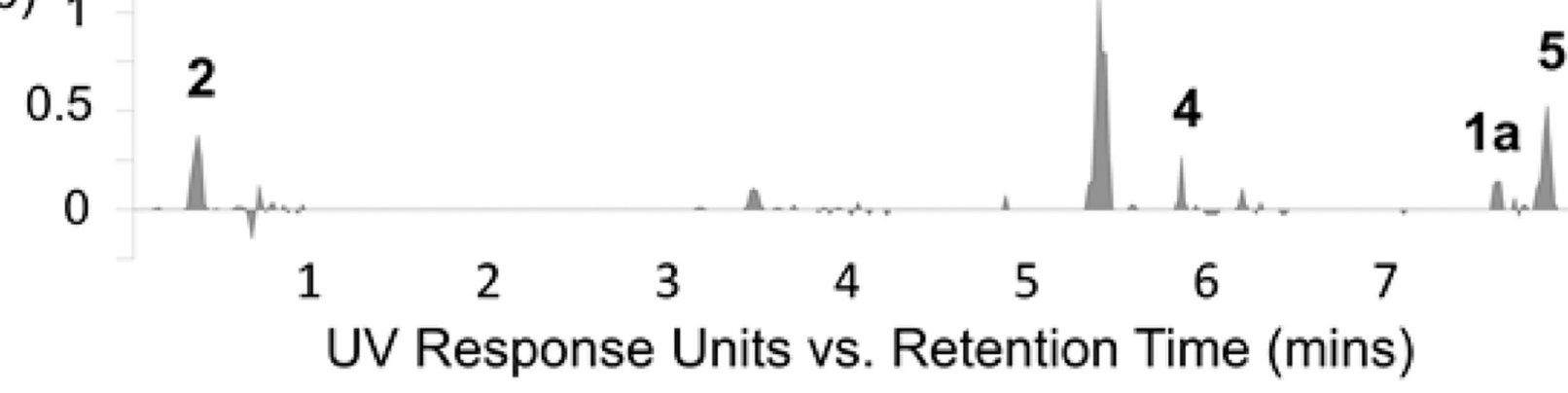<smiles></smiles>

(1a) DMNB-Phosphine $\mathrm{RT}=7.62$<smiles>CC(CC#N)C(=O)[O-]</smiles>

(2) Azidohomolanine $\mathrm{RT}=0.43$<smiles>NC(CCNC(=O)c1ccccc1P(=O)=O)C(=O)[O-]</smiles>

(3) Ligated Product $\mathrm{RT}=5.34$<smiles>COC(=O)c1ccccc1P</smiles>

(4) Free Staudinger Phosphine $\mathrm{RT}=5.86$<smiles>COC(=O)c1ccccc1P(=O)(O)c1ccccc1</smiles>

(5) Staudinger Phosphine Oxide $\mathrm{RT}=7.92$

Figure 1.

Determination of 1a uncaging efficiency. An initial sample containing $6.5 \mathrm{mM} 1 \mathrm{a}$ and 10.0 mM 2 was irradiated with UV light ( $350 \mathrm{~W}$ medium pressure $\mathrm{Hg}$ arc lamp) and left unperturbed for $6 \mathrm{~h}$. LC-UV-MS analysis of samples before (a) and after (b) light exposure facilitated identification and quantification of products formed (5-95\% acetonitrile over 20 $\min )$. An internal standard of triphenylphosphine oxide was used for quantification purposes (not shown). 
a)<smiles>N#CCNC1CC(O)(C(=O)[O-])CC(O)C1CC(O)CO</smiles><smiles>[R]c1ccc(C(=O)OC)c([PH2]([Y20])C)c1</smiles>
UV Light

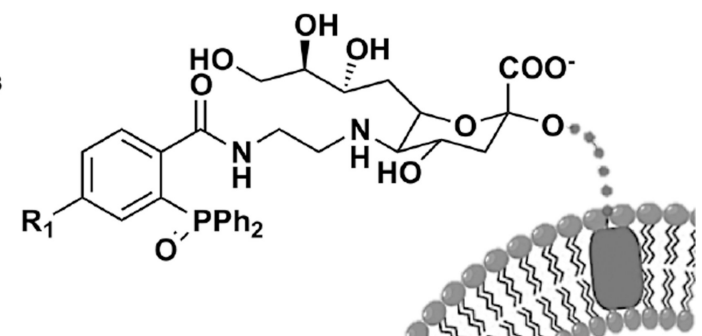

1a: $R_{1}=H, X=D M N B$

1b: $R_{1}=$ DYKDDDDK, $X=D M N B$

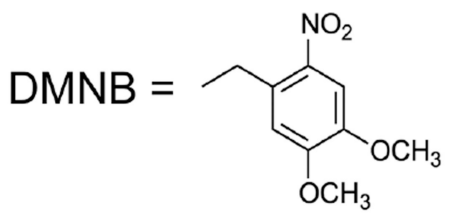

b)

$\mathrm{Ac}_{4} \operatorname{ManNAz}$ UV exposure time (min) 1b

\section{Phosphine-FLAG}

\section{$175 \mathrm{kDa}$

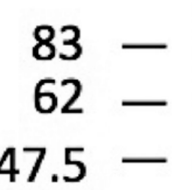 \\ $32.5-$ $25-$}

Figure 2.

(a) Biomolecular tagging by photocaged Staudinger-Bertozzi phosphines and (b) Western blot analysis of glycoprotein labeling by $\mathbf{1 b}$. (a) Glycoproteins modified with an azidosugar can be incubated with a reporter-conjugated, photocaged Staudinger-Bertozzi phosphine in the presence of UV light ( $350 \mathrm{~W}$ medium pressure $\mathrm{Hg}$ arc lamp) to yield a tagged biomolecule. (b) HEK293 cells were cultured in the presence of $50 \mu \mathrm{M} \mathrm{Ac} 4$ ManNAz for 3 days and subsequently lysed. HEK293 cells cultured in the absence of azidosugar were used as a negative control. Staudinger ligation time is $3 \mathrm{~h}$. Whole-cell lysates were incubated with 
$500 \mu \mathrm{M}$ of $\mathbf{1 b}$ or phosphine-FLAG as a control. Samples were separated by SDS-PAGE and immunostained with an anti-FLAG M2-HRP antibody. 
a)
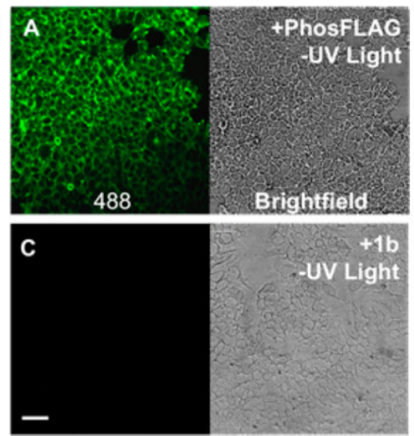

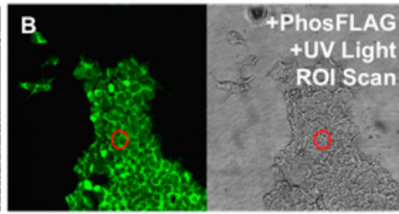

D b)

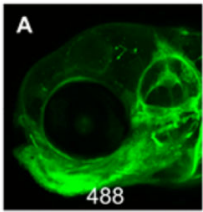

+uv Light Rolscan

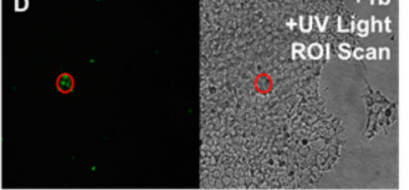

c

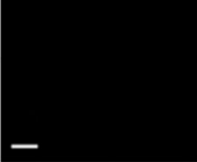

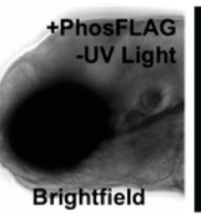

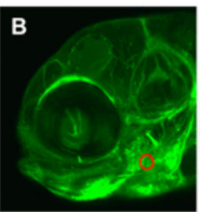

D

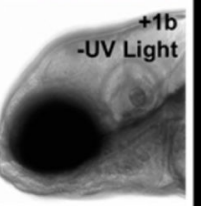

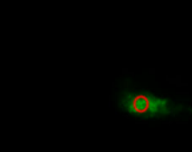

+PhosFLAG +UV Light rol Sc (1) .

Figure 3.

Fluorescence microscopy images of cell-surface azidoglycoproteins spatially labeled by $\mathbf{1 b}$ (a) on fixed cells and (b) on fixed zebrafish. (a) Single $z$-plane fluorescence images of HEK293 cells. HEK293 cells were cultured in the presence of $50 \mu \mathrm{M} \mathrm{Ac} 4 \mathrm{ManNAz}$ for 3 days and subsequently fixed with methanol. Cells cultured in the absence of sugar were used as a negative control (Figure S4). Cells were incubated with $300 \mu \mathrm{M}$ of $\mathbf{1 b}$ or phosphineFLAG as a control. +UV samples were irradiated using a laser scanning confocal microscope (Leica SP5 405 diode, <1 min) and subsequently left unperturbed for $1 \mathrm{~h}$ after irradiation. Cells were then immunostained with an anti-FLAG-FITC antibody and thereafter visualized. Scale bar represents $50 \mu \mathrm{m}$. Data are representative of experiments performed in triplicate. (b) $z$-Projection fluorescence images of zebrafish head region. Zebrafish embryos were injected with a $5 \mathrm{~nL}$ solution containing $5 \mathrm{mM}$ GalNAz and left to develop to $72 \mathrm{~h}$ postfertilization (hpf). Embryos injected with vehicle alone were used as a negative control (Figure S5). Partially mounted embryos were then treated with $\mathbf{1 b}$ or phosphine-FLAG as a control. +UV samples were irradiated using a laser scanning confocal microscope (Leica SP5 405 diode, <1 min) and subsequently left unperturbed for $1 \mathrm{~h}$ after irradiation. Embryos were then fixed and immunostained with an anti-FLAG-FITC antibody. Scale bar represents $100 \mu \mathrm{m}$. Regions of interest (red circles) define the spatially localized irradiation area. Data are representative of experiments performed in triplicate. 